of helioseismology together should go a long way towards clearing up these questions.

In view of all this, the three volumes of The Physics of the Sun have made a timely appearance, in an appropriate form for the physicist. Not only do the 31 authors of the 22 chapters review the present state of knowledge, but they press on to consider the outstanding puzzles and dilemmas, summarized in the introductory chapter. For that reason the books will serve as a basic reference source for the next decade, and perhaps beyond.

It will be interesting to look back from the anticipated wisdom of the 1990 s to see if the resolution of the neutrino discrepancy lies in an error in the thermonuclear reaction rates (reviewed in Chapter 2), or in the radiative transport in solar matter (Chapter 3) or perhaps in some unexpected mixing in the central regions (Chapter 4), which may in turn be related to the turbulent circulation in the convective zone (Chapter 5) and internal stresses (Chapter 6). In all probability, however, these questions cannot be resolved without an understanding of the structure of the solar interior to be derived from solar oscillations in the manner described in Chapter 7.

Essentially, all information concerning the activity and composition of the Sun comes from observations of the solar atmosphere, reviewed succinctly in Chapter 8 . The active suprathermal atmosphere is described in Chapters 9 and 10 , and the dynamics of magnetic fields and fluids responsible for the activity in Chapters 11 and 12. The acceleration of fast particles and the associated nuclear reactions in flares are reviewed in Chapters 13 and 14, with the radio emission from the many forms of outburst treated in Chapter 15.

Chapter 16 reviews present facts and ideas about the formation of the Sun and planets. Chapter 17 provides a critical discussion of the problem posed by the low neutrino flux, with an examination of some of the possible causes. Chapter 18 treats the solar wind phenomenon in the broad context of all stars, with coronae and winds. The magnetic activity that drives the suprathermal atmosphere of the Sun and other stars is covered in Chapter 19 , while the last three contributions review the effects of the hard radiation, the solar wind and the fast particles on the terrestrial environment.

There has been no more vivid portrayal of the diverse and exotic phenomena that make up a "pedestrian" star like the Sun. One needs only to be close enough to see the details to discover, and to be challenged by, the physics of our nearest star.

Eugene N. Parker is a Professor in the Department of Physics, University of Chicago, Chicago, Illinois 60637, USA.

\section{Picture histology}

Nancy J. Lane

A Colour Atlas of Insect Tissues via the Flea. By Miriam Rothschild, Yosef Schlein and Susumo Ito. Wolfe Science, London/Methuen, New York: 1986. Pp. 184. £40, $\$ 60$.

THIs large, well-bound volume is intended to fill the gap between two earlier books, one on the general anatomy of insect tissues and the other on the fine structure of individual insect cells. It contains numerous colour micrographs of sectioned histological preparations from the various organs of the flea; together with higher resolution electron micrographs of many of the same tissues, these splendid plates make up the bulk of the book. A series of beautiful line drawings preface the plates and illustrate the flea's principal internal organs. As a guide to the histology of insect tissues this is an admirable compendium since it deals with all the component organs, and for each there is a separate foreword describing its organization and function.

In their introductory remarks, the authors express the hope that the colour micrographs will "stir the imagination and excite the aesthetic susceptibilities" of students. This they almost certainly will do, as may the cover, a particularly striking multi-coloured histological section of a gravid flea's oviduct and vagina!

The rationale for the Atlas is to illustrate the cellular organization of the tissues of all insects, using the flea as a typical example. The choice of organism was appropriate because the flea's small size allows the cutting and mounting of sections through the entire animal, so that many organs can be observed at once. Moreover, as an ectoparasite and potential carrier of bubonic plague, myxomato- sis and typhus, the flea is a good example of a vector of arthropod-borne diseases of mammals. As such it has certain peculiarities, and at the same time lacks other features, but an appendix illustrates some of these latter structures from other insects.

There are a few inaccuracies - such as the mislabelling of what is probably a gap junction as a tight junction (these are only present in certain tissues in insects), and in the definition of "perineurium" (which consists of the glial cells but not the connective tissue surrounding nerves and ganglia) - and a degree of repetition in some illustrations; at times, one finds oneself wishing that the second of two rather similar micrographs was at a higher power to resolve more detail. Some tissues are heavily illustrated, whereas others, for example the dorsal vessel, have only one accompanying light micrograph. There is an inconsistency, too, as to whether or not (or indeed how many) electron micrographs of each tissue are provided; some, such as the sensilium and the unfortunate dorsal vessel, have none! A few more specialized references would also have been welcome for those not so well-versed in the subject as the authors.

The book will undoubtedly be extremely valuable to the novice, and will also serve as a practical guide for established entomologists wishing to examine organs with which they are unfamiliar; conveniently, it is bound so as to remain flat when opened for study. It is likely to be widely used and a copy ought to be available in every department that actively pursues insect physiology. That it also has the glossy appearance of a coffee-table text need not detract from its usefulness and practicality in the laboratory.

Nancy $J$. Lane is in the Agriculture and Food Research Council's Unit of Insect Neurophysiology and Pharmacology, Department of Zoology, University of Cambridge, Downing Street, Cambridge CB2 $3 E J, U K$.

\section{IMAGE \\ UNAVAILABLE FOR COPYRIGHT REASONS}

All steamed up - geyser activity near Healdsburg, California, $100 \mathrm{~km}$ north-west of San Francisco. The picture is reproduced from The Geology of Ore Deposits by John M. Guilbert and Charles F. Park, Jr, published earlier this year by W.H. Freeman. 